\title{
Gastric gastrointestinal stromal tumors: clinical features and short- and long-term outcomes of laparoscopic resection
}

\author{
Maciej Stanek ${ }^{1,2}$, Magdalena Pisarska ${ }^{1,2}$, Dorota Budzyńska ${ }^{1,2}$, Anna Rzepa ${ }^{1}$, Michał Pędziwiatr ${ }^{1,2}$, Piotr Major ${ }^{1,2}$, \\ Andrzej Budzyński ${ }^{1,2}$ \\ ${ }^{1} 2^{\text {nd }}$ Department of General Surgery, Jagiellonian University Medical College, Krakow, Poland \\ ${ }^{2}$ Department of Endoscopic, Metabolic and Soft Tissue Tumor Surgery, Jagiellonian University Medical College, Krakow, Poland \\ Videosurgery Miniinv 2019; 14 (2): 176-181 \\ DOI: https://doi.org/10.5114/wiitm.2019.83868
}

\begin{abstract}
Introduction: Although minimally invasive techniques are currently recognized as effective and validated treatment for small gastric gastrointestinal stromal tumors (GISTS), the role of laparoscopy is not yet established, especially in the institutions that have less experience in minimally invasive surgery.

Aim: To evaluate the outcomes of laparoscopic treatment of gastric gastrointestinal stromal tumors compared to the results obtained in a group of patients treated with conventional surgery.

Material and methods: A retrospective analysis of data collected for a group of 68 patients treated for gastric GIST in the period from 2002 to 2017 was performed. Forty-six patients were treated laparoscopically (group 1) and 22 patients underwent conventional surgery (group 2). The analyzed medical data included clinical and pathomorphological features of removed tumors, perioperative parameters as well as short and long-term results of surgical treatment.

Results: Histopathological examination confirmed radical resection for all patients. No deaths were reported in the 30-day post-operative period. Patients in group 1 had significantly shorter length of hospital stay ( 3 vs. 9 days), less intra-operative blood loss (25 vs. $175 \mathrm{ml}$ ) and fewer perioperative complications (13\% vs. 41\%) compared to group 2. The mean post-operative follow-up was 57 months. During this period, four patients died for reasons unrelated to the primary disease. None of the patients who underwent a laparoscopic procedure had a recurrence of the tumor in the follow-up period.

Conclusions: Laparoscopy in the treatment of gastric GISTs has unquestionable advantages, including decreased blood loss, reduced risk of complications, and shorter hospital stay.
\end{abstract}

Key words: laparoscopy, gastric gastrointestinal stromal tumor, gastric wedge resection.

\section{Introduction}

Gastrointestinal stromal tumors (GISTs) were first classified as a distinct type of neoplasm in 1983 [1]. Although they are relatively rare, they are considered the most prevalent mesenchymal neoplasms of the digestive tract [2]. Their most common location is the stomach, but they can also occur in other parts of the digestive system as well as outside of it (urinary bladder, extraperitoneal space) [3]. They are characterized by considerable clinical diversity, ranging from small, localized, slow-growing lesions to very aggressive tumors that already have massive metastases upon diagnosis [4, 5].

The treatment of initially resectable tumors involves their removal with a margin of the surround-

\section{Address for correspondence}

Piotr Major MD, PhD, $2^{\text {nd }}$ Department of General Surgery, Jagiellonian University Medical College, 21 Kopernika St, 31-501 Krakow, Poland, phone: +48 693313 948, e-mail: majorpiotr@gmail.com 
ing healthy tissue [6]. The removal of the regional lymphatic tissue is not required, as metastasis of GISTs to lymph nodes is rarely observed [7].

In the last three decades, laparoscopy has played an increasingly important role in the treatment of both benign and malignant tumors. The first report about use of this approach for removal of a gastric GIST comes from 1992 [8]. It was a small tumor with very low potential for malignancy. In the following years, the treatment of these tumors by laparoscopy gained increasing support. Although minimally invasive techniques are currently recognized as effective and validated treatment for small gastric GIST, the role of laparoscopy is not yet established, especially in the institutions that have less experience in minimally invasive surgery [9].

\section{Aim}

The aim of the study was to evaluate the outcomes of laparoscopic treatment of gastric gastrointestinal stromal tumors compared to the results obtained in a group of patients treated with conventional surgery.

\section{Material and methods}

\section{Design}

A retrospective analysis of data collected for a group of patients treated for gastric GIST at the $2^{\text {nd }}$ Department of General Surgery at the Jagiellonian University in Krakow in the period from 2002 to 2017 was performed. The study group included patients with an initially resectable tumor that was confirmed to be a gastric stromal neoplasm upon histopathological examination of the surgical specimen. Patients who underwent neoadjuvant treatment with tyrosine kinase inhibitors, those who at the time of the diagnosis had distant metastases, and patients with an additional malignant neoplasm were excluded from the study.

The analyzed medical data included clinical and pathomorphological features of removed tumors, perioperative parameters as well as short- and longterm results of surgical treatment.

Patients were divided into two groups - those who were treated laparoscopically (group 1) and patients who underwent conventional surgery (group 2). Factors affecting the short- and long-term outcomes were compared between the two groups.
During the pre-operative period, a computed tomography (CT) scan and a gastroscopy with a sample for histopathological examination were performed for each patient. The tumors' potential for malignancy was determined according to the ESMO 2012 guidelines. The largest dimension of the GIST measured during the histopathological examination was recorded as the tumor's diameter. Blood loss and operative time were established on the basis of documentation prepared by surgeons and anesthesiologists participating in the operation. Surgery-related risk was estimated using the American Society of Anesthesiologists (ASA) scale, while the post-operative complications were evaluated according to the Clavien-Dindo classification [10].

\section{Material}

In the analyzed period, 68 patients (47 women and 21 men) were treated for gastric GISTs at the $2^{\text {nd }}$ Department of General Surgery at the Jagiellonian University in Krakow. The mean age of the patients was 63.75 years.

Laparoscopy was performed for 46 of the patients. The remaining 22 patients underwent conventional surgery (these patients were operated on in the first few years of the analyzed period). The demographics of the patients are presented in Table I, whereas the type of surgery is shown in Table II. Only the tumor characteristics according to the ESMO classification including size were significantly different.

\section{Ethics}

The study was approved by the Ethics Committee of the Jagiellonian University (KBET/1072. 6120.117 .2018 ). All procedures were performed in accordance with the latest revision of the Declaration of Helsinki.

\section{Statistical analysis}

All data were analyzed with StatSoft Statistica v.13. The results are presented as mean \pm standard deviation (SD), median and interquartile range (IQR) when appropriate. For the purposes of further analysis, the entire group of patients was divided into two groups. The study of categorical variables used the $\chi^{2}$ test of independence. The Shapiro-Wilk test was used to check for normal distribution of data and Student's $t$-test was used for normally distributed quantitative data. For non-normally distributed quantitative vari- 
Table I. Demographics of patients

\begin{tabular}{|c|c|c|c|c|}
\hline Parameter & $\begin{array}{l}\text { Total } \\
n(\%)\end{array}$ & $\begin{array}{c}\text { Group } 1 \\
n(\%)\end{array}$ & $\begin{array}{c}\text { Group } 2 \\
n(\%)\end{array}$ & $P$-value \\
\hline Number of patients & 68 & 46 & 22 & - \\
\hline Females, $n(\%)$ & $47(69.1)$ & $33(71.7)$ & $14(63.6)$ & 0.4987 \\
\hline Males, $n(\%)$ & $21(30.9)$ & $13(28.3)$ & $8(36.4)$ & \\
\hline Age, median (IQR) & $66.5(55.5-74.5)$ & $67(59-75)$ & $65(55-70)$ & 0.5597 \\
\hline BMI, median (IQR) & $27.7(25.2-32.4)$ & $27.8(23.9-33.1)$ & $27.2(26.2-31.2)$ & 0.9802 \\
\hline ASA $1, n(\%)$ & $1(1.5)$ & $1(2.2)$ & - & 0.0276 \\
\hline ASA $2, n(\%)$ & $42(61.8)$ & $33(71.7)$ & $9(40.9)$ & \\
\hline ASA 3, $n(\%)$ & $25(36.7)$ & $12(26.1)$ & $13(59.1)$ & \\
\hline Location, $n(\%)$ : & & & & 0.6814 \\
\hline Cardia & $11(16.2)$ & $6(13.0)$ & $5(22.7)$ & \\
\hline Lesser curvature & $15(22.1)$ & $10(21.7)$ & $5(22.7)$ & \\
\hline Greater curvature & $34(50.0)$ & $25(54.4)$ & $9(40.9)$ & \\
\hline Antrum & $8(11.7)$ & $5(10.9)$ & $3(13.7)$ & \\
\hline Histological subtypes, $n$ (\%): & & & & 0.6613 \\
\hline Spindle cell & $46(67.6)$ & $30(65.2)$ & $16(72.7)$ & \\
\hline Epithelioid & $10(14.7)$ & $8(17.4)$ & $2(9.1)$ & \\
\hline Mixed & $12(17.6)$ & $8(17.4)$ & $4(18.2)$ & \\
\hline ESMO risk classification, $n(\%)$ : & & & & 0.0326 \\
\hline Very low & $14(20.6)$ & $11(23.9)$ & $3(13.6)$ & \\
\hline Low & $34(50.0)$ & $23(50.0)$ & $11(50)$ & \\
\hline Moderate & $12(17.6)$ & $10(21.7)$ & $2(9.1)$ & \\
\hline High & $8(11.8)$ & $2(4.4)$ & $6(27.3)$ & \\
\hline Diameter, median (IQR) $[\mathrm{cm}]$ & $4.35(3.55-5.5)$ & $4(3.5-5.5)$ & $4.75(4-7)$ & 0.0319 \\
\hline
\end{tabular}

Table II. Type of surgery

\begin{tabular}{|ll|}
\hline Laparoscopic treatment (group 1): \\
\hline Wedge gastric resection using stapler & 19 \\
\hline Local transmural gastrectomy & 27 \\
\hline Open treatment (group 2): & \\
\hline Wedge gastric resection using stapler & 9 \\
\hline Local transmural gastrectomy & 7 \\
\hline Distal gastrectomy & 4 \\
\hline Totaximal gastrectomy & 1 \\
\hline
\end{tabular}

ables, the Mann-Whitney $U$ test was used. Results were considered statistically significant when the $p$-value was found to be less than 0.05 .

\section{Results}

No intra-operative tumor ruptures were reported regardless of the study group, and the histopathological examination confirmed radical resection (RO margins) for all patients. The clinical and pathomorphological features of the removed tumors are shown in Table III.

None of the patients who underwent laparoscopic treatment required subsequent open surgery. No deaths were reported in the 30-day post-operative 
period. Patients in group 1 (treated laparoscopically) had a significantly shorter length of hospital stay, less intra-operative blood loss, and fewer perioperative complications (13\% vs. $41 \%$ ) compared to group 2 (those who underwent open surgery). Short-term outcomes of treatment are shown in Table IV. Two patients required reoperation (both from group 1 ). In one of these patients, bleeding from the short gastric vessels was observed in the postoperative period. The other patient had symptoms of obstruction of the gastrointestinal tract due to narrowing of the stomach by the staple line at the location of the tumor. The remaining complications in either study group were not sufficiently serious to require surgical intervention (grade I and II in the Clavien-Dindo classification). Two patients from group 1 were rehospitalized - one due to suspected bleeding from the upper gastrointestinal tract (eventually ruled out), and another due to a small fluid collection in the vicinity of the stomach in the post-operative period (managed conservatively).

The mean post-operative follow-up for all patients in the study was 57 months (6-160 months). During this period, 4 patients ( 3 from group 1 and 1 from group 2) died for reasons unrelated to the primary disease. None of the patients who underwent a laparoscopic procedure received adjuvant treatment with tyrosine kinase inhibitors in the postoperative period, and none of them experienced any symptoms indicating the recurrence of the tumor in the follow-up period. In the group treated via open surgery, 5 patients were diagnosed with metastatic liver tumors (median time before recurrence was 22 months). These patients were treated with imatinib. During this treatment, no progression of the condition was observed for any of the patients.

\section{Discussion}

The comparison of post-operative data for patients with gastric GISTs showed that laparoscopic

Table III. Clinical and pathomorphological features of the removed tumor

\begin{tabular}{|c|c|}
\hline Parameter & $N(\%)$ \\
\hline \multicolumn{2}{|l|}{ Symptoms: } \\
\hline Pain & $25(36.8)$ \\
\hline Nausea & $10(14.7)$ \\
\hline Vomiting & $2(2.9)$ \\
\hline Weight loss & $5(7.4)$ \\
\hline Heartburn & $3(4.4)$ \\
\hline Bleeding of $\mathrm{Gl}$ tract & $18(26.5)$ \\
\hline Asymptomatic & $15(22.1)$ \\
\hline \multicolumn{2}{|c|}{ Immunohistochemical phenotype: } \\
\hline CD $117+$ & $66(97.1)$ \\
\hline CD $34+$ & $48(70.6)$ \\
\hline DOG1 + & $18(26.5)$ \\
\hline SMA + & $6(8.8)$ \\
\hline Desmin + & $5(7.4)$ \\
\hline $\mathrm{S} 100+$ & $3(4.4)$ \\
\hline PKC theta + & $1(1.5)$ \\
\hline \multicolumn{2}{|l|}{ Size: } \\
\hline Tumor $\leq 5 \mathrm{~cm}$ & $46(67.6)$ \\
\hline Tumor $>5 \mathrm{~cm}$ & $22(32.4)$ \\
\hline \multicolumn{2}{|c|}{ GISTs confirmed before operation: } \\
\hline Yes & $20(29.4)$ \\
\hline No & $48(70.6)$ \\
\hline
\end{tabular}

Table IV. Short-term outcomes of treatment

\begin{tabular}{|c|c|c|c|}
\hline Parameter & Group 1 & Group 2 & $P$-value \\
\hline Length of hospital stay, median (IQR) [days] & $3(3-5)$ & $9(8-11)$ & $<0.0001$ \\
\hline Operative time, median (IQR) & $100(70-120)$ & $95(80-150)$ & 0.1934 \\
\hline Intraoperative blood loss, median (IQR) & $25(15-50)$ & $175(100-205)$ & $<0.0001$ \\
\hline Patients without complications, $n(\%)$ & $40(87.0)$ & $13(59)$ & 0.0034 \\
\hline Clavien-Dindo $1, n(\%)$ & $3(6.5)$ & $2(9.1)$ & \\
\hline Clavien-Dindo $2, n(\%)$ & $1(2.2)$ & $7(31.9)$ & \\
\hline Clavien-Dindo 3, $n$ (\%) & $2(4.3)$ & - & \\
\hline
\end{tabular}


surgery was associated with significantly less blood loss, shorter hospitalization time, and a lower number of complications. Although nearly one third of the tumors were larger than $5 \mathrm{~cm}$, both laparoscopic and conventional surgery resulted in radical resection, as confirmed by macroscopic assessment and histological examination.

The term gastrointestinal stromal tumors (GISTs) was introduced in 1983 by Mazur and Clark [1]. They originate from the precursors of cells of Cajal [11]. They are typically characterized by the expression of CD117, which is observed for $95 \%$ of these tumors. Other markers associated with GISTs include CD34, DOG1 and - less frequently - S100, SMA or desmin [12]. In the present study, CD117 expression was observed for $97.1 \%$ of the patients, while CD34 expression was found for $70.1 \%$ of them. The expression of the remaining markers was much less frequent. As per the current guidelines, the determination of these markers is key to the proper diagnosis of GISTs. In questionable cases, it is recommended that the KIT and PDGFRA genes be checked for mutation; this is especially significant in the case of GIST tissue negative for CD117 [9]. It should be emphasized that genetic examinations have an increasingly important role in diagnostics and treatment of many types of neoplasms [13].

Gastrointestinal stromal tumors can be excised with a small margin of healthy tissue without lymphadenectomy because they infrequently metastasize to lymph nodes (fewer than $3 \%$ of cases based on the available literature data $[6,7])$. This makes minimally invasive techniques particularly suitable for the removal of GISTs, especially gastric ones. The first procedure of this type was performed more than 25 years ago [8]. Laparoscopy is currently a well-established method of treatment of small gastric GISTs [9]. There is a widespread opinion that if the team has adequate experience and will exercise sufficient care to maintain sufficient tumor-free margins during resection, the size of the tumor itself does not exclude the use of minimally invasive procedures $[14,15]$. Its advantages over open surgery, including a lower post-operative pain level, earlier mobilization of the patient and shorter length of hospital stay, are well known [16]. This also applies to the patients in the present study, in whom laparoscopy was associated with a shorter hospital stay and reduced intra-operative blood loss. Similar observations were also made by other authors $[17,18]$.
When analyzing the results of laparoscopic treatment, the number and type of complications seem to be key factors. According to the literature, complication rates for this method range from several to a dozen or so percent, pulmonary complications (pneumonia, pulmonary embolism) being the most frequently reported type $[3,17]$. In the present study, perioperative complications were reported for $13 \%$ of the patients who underwent laparoscopic surgery (group 1). This rate was significantly lower than in the group treated via open surgery (41\%). It should be emphasized that the vast majority of all complications were grade I and II in the Clavien-Dindo classification and did not require surgical intervention; only two patients from group 1 had grade III complications and required reoperation. However, it is important to underline that tumors removed in patients from group 2 were larger and had higher potential for malignancy, which might have affected the complication rate in this group.

In the case of GIST removal, the rate of conversion from laparoscopy to laparotomy appears to be low. In their paper, Roggin and Posner present a review of results from five reports on the laparoscopic treatment of GISTs. According to this source, the conversion rates range from 0 to $6.5 \%$ [3]. In the present study, not a single case of conversion to laparotomy was found. At the same time, no intra-operative tumor rupture occurred, a noteworthy result given the fact that tumor rupture is a very significant prognostic factor for the recurrence of GIST. It appears that this complication can be avoided provided that the appropriate surgical technique is applied and the tissues are dissected in a sufficiently careful manner [19].

The analysis of a significant amount of data revealed no statistically significant differences in the 5 -year survival rates of patients treated with either of these techniques $[20,21]$. In the present study, the median post-surgery follow-up was just under 5 years. The survival rate in the group treated via laparoscopy was $93.4 \%$. None of the three deaths in the study group was due to neoplasm. Kim et al. reported a 5-year survival rate of $98.6 \%$ and $94.8 \%$ of patients without progression of the disease. None of the patients who underwent laparoscopic surgery received adjuvant treatment with tyrosine kinase inhibitors, and no recurrences were observed. Adjuvant therapy with imatinib is recommended in the case of GISTs with a very high risk of recurrence $[9,22]$. 
Two patients treated laparoscopically met inclusion criteria for adjuvant treatment, but did not receive it due to advanced age and comorbidities. These patients died during the follow-up period for reasons unrelated to the progression of GIST. In the group analyzed in this study, GIST metastasized to the liver in five patients treated with open surgery (incidence of $22.7 \%$ ). In all these patients GIST had a high risk of relapse, which was more frequently observed in the group of patients undergoing laparotomy. All these patients received imatinib after the diagnosis of progression and had stable disease at the end of the 5-year follow-up.

\section{Conclusions}

Laparoscopy in the treatment of gastric GIST has similar efficacy as the classical open surgery. Minimally invasive procedures have unquestionable advantages, including decreased blood loss, reduced risk of complications, and shorter hospital stay. The team's experience seems to be the most important factor reducing the incidence of complications and enabling safe extension of the use of laparoscopy to the treatment of oncological patients.

\section{Conflict of interest}

The authors declare no conflict of interest.

\section{References}

1. Mazur MT, Clark HB. Gastric stromal tumors. Reappraisal of histogenesis. Am J Surg Pathol 1983; 7: 507-19.

2. Lim KT, Tan KY. Current research and treatment for gastrointestinal stromal tumors. World J Gastroenterol 2017; 23: 4856-66.

3. Roggin KK, Posner MC. Modern treatment of gastric gastrointestinal stromal tumors. World J Gastroenterol 2012; 18: 6720-8.

4. Al-Kalaawy M, El-Zohairy MA, Mostafa A, et al. Gastrointestinal stromal tumors (GISTs), 10-year experience: patterns of failure and prognostic factors for survival of 127 patients. J Egypt Natl Canc Inst 2012; 24: 31-9.

5. Walędziak M, Różańska-Walędziak A, Kowalewski PK, et al. Bariatric surgery and incidental gastrointestinal stromal tumors a single-center study: VSJ Competition, 1(st) place. Videosurgery Miniinv 2017; 12: 325-9.

6. Zhao X, Yue C. Gastrointestinal stromal tumor. J Gastrointest Oncol 2012; 3: 189-208.

7. Sicklick JK, Lopez NE. Optimizing surgical and imatinib therapy for the treatment of gastrointestinal stromal tumors. J Gastrointest Surg 2013; 17: 1997-2006.

8. Lukaszczyk JJ, Preletz RJ Jr. Laparoscopic resection of benign stromal tumor of the stomach. J Laparoendosc Surg 1992; 2: 331-4.
9. ESMO/European Sarcoma Network Working Group. Gastrointestinal stromal tumours: ESMO Clinical Practice Guidelines for diagnosis, treatment and follow-up. Ann Oncol 2014; 25 Suppl 3: iii21-6.

10. Clavien PA, Barkun J, de Oliveira ML, et al. The Clavien-Dindo classification of surgical complications: five-year experience. Ann Surg 2009; 250: 187-96.

11. Miettinen M, Lasota J. Gastrointestinal stromal tumors - definition, clinical, histological, immunohistochemical, and molecular genetic features and differential diagnosis. Virchows Arch 2001; 438: 1-12.

12. Rubin BP, Blanke CD, Demetri GD, et al. Protocol for the examination of specimens from patients with gastrointestinal stromal tumor. Arch Pathol Lab Med 2010; 134: 165-70.

13. Szeliga J, Sondka Z, Jackowski M, et al. NOD2/CARD15 polymorphism in patients with rectal cancer. Med Sci Monit 2008; 14: CR480-4.

14. Smolarek S, Pomeroy E, Kinnarney F, et al. Laparoscopic resection of large gastric gastrointestinal stromal tumours. Videosurgery Miniinv 2016; 11: 31-7.

15. Kermansaravi M, Rokhgireh S, Darabi S, et al. Laparoscopic total gastrectomy for a giant gastrointestinal stromal tumor (GIST) with acute massive gastrointestinal bleeding: a case report. Videosurgery Miniinv 2017; 12: 306-10.

16. Kinoshita T, Shibasaki H, Oshiro T, et al. Comparison of laparoscopy-assisted and total laparoscopic Billroth-I gastrectomy for gastric cancer: a report of short-term outcomes. Surg Endosc 2011; 25: 1395-401.

17. Chen QF, Huang CM, Lin M, et al. Short- and long-term outcomes of laparoscopic versus open resection for gastric gastrointestinal stromal tumors: a propensity score-matching analysis. Medicine (Baltimore) 2016; 95: e3135.

18. Goh BK, Goh YC, Eng AK, et al. Outcome after laparoscopic versus open wedge resection for suspected gastric gastrointestinal stromal tumors: a matched-pair case-control study. Eur J Surg Oncol 2015; 41: 905-10.

19. Milone M, Elmore U, Musella M, et al. Safety and efficacy of laparoscopic wedge gastrectomy for large gastrointestinal stromal tumors. Eur I Surg Oncol 2017; 43: 796-800.

20. Kim KH, Kim MC, Jung GJ, et al. Long term survival results for gastric GIST: is laparoscopic surgery for large gastric GIST feasible? World J Surg Oncol 2012; 10: 230.

21. Ye L, Wu X, Wu T, et al. Meta-analysis of laparoscopic vs. open resection of gastric gastrointestinal stromal tumors. PLoS One 2017; 12: e0177193.

22. Rutkowski P, Gronchi A. Efficacy and economic value of adjuvant imatinib for gastrointestinal stromal tumors. Oncologist 2013; 18: 689-96.

Received: 27.02.2019, accepted: 2.03.2019. 